\title{
Remark on approximation in the calculation of the primordial spectrum generated during inflation
}

\author{
D.H. Huang ${ }^{1}$, W.B. Lin ${ }^{2}$, X.M. Zhang ${ }^{3,2}$ \\ ${ }^{1}$ Deptartment of Physics, Peking University, Beijing 100871, P.R. China; \\ 2 Institue of High Energy Physics, Chinese Academy of Science, P.O. Box 918-4, Beijing 100039, \\ P.R. China; \\ ${ }^{3}$ CCAST (World Lab), PO Box 8370, Beijing 100080, P. R. China
}

\begin{abstract}
We re-examine approximations in the analytical calculation of the primordial spectrum of cosmological perturbation produced during inflation. Taking two inflation models (chaotic inflation and natural inflation) as examples, we numerically verify the accuracy of these approximations.
\end{abstract}

Typeset using REVTEX 
The inflationary prediction on the primordial power spectrum of the cosmological perturbation is testable by the observations of the large-scale structures [1], therefore it is important to make the prediction more precise. In this brief report, we re-examine the analytical calculation of the primordial spectrum of cosmological perturbation produced during inflation and point out a subtlety in the approximations.

To begin with, we review briefly the derivation of the power spectrum using the standard slow rolling approximation (SRA) technique [2,3].

The most general form of the metric for the background and the scalar metric perturbations can be written as 《4

$$
d s^{2}=a^{2}(\eta)\left\{(1+A) d \eta^{2}-2 \partial_{i} B d x^{i} d \eta-\left[(1-2 \Psi) \delta_{i j}+2 \partial_{i} \partial_{j} E\right] d x^{i} d x^{j}\right\}
$$

where $\eta \equiv \int d t / a(t)$ is conformal time.

The primordial spectrum of scalar perturbation is defined as

$$
P_{S}(k) \equiv \frac{k^{3}}{2 \pi^{2}}\left|\mathcal{R}_{k}\right|^{2}
$$

where $\mathcal{R}_{k}$ is the coefficient of the Fourier transformation of the intrinsic curvature perturbation of comoving hypersurfaces $\mathcal{R}$ which during inflation has the form [3, [0]

$$
\mathcal{R} \equiv-\Psi-\frac{H}{\dot{\phi}} \delta \phi
$$

where $\delta \phi$ represents the fluctuation of the inflaton field $\phi$ and $H=\dot{a} / a$ is the Hubble constant.

Note that $P_{S}(k)$ in Eq.(2) by definition is constant, however, as we can see later the power spectrum during the period of inflation evolves in time and approaches a constant only when $k / a H \rightarrow 0$. For the convenience of our discussion we introduce $P_{S}(k, a)$, the power spectrum of a given $k$ mode as a function of time, to distinguish from its asymptotic constant value $\left.P_{S}(k) \equiv P_{S}(k, a)\right|_{k / a H \rightarrow 0}$.

To calculate $P_{S}(k, a)$, one usually defines $u_{k} \equiv-z \mathcal{R}_{k}$ where $z \equiv a \dot{\phi} / H$. And $u_{k}$ satisfies the following equation of motion [6] 


$$
u_{k}^{\prime \prime}+\left(k^{2}-\frac{z^{\prime \prime}}{z}\right) u_{k}=0
$$

where a prime denotes the derivative with respect to conformal time $\eta$.

According to the flat spacetime field theory (well inside the horizon), one has

$$
u_{k} \rightarrow \frac{1}{\sqrt{2 k}} e^{-i k \eta} \quad \text { as } \quad k / a H \rightarrow \infty
$$

On the other hand, the solution of Eq.(4) in the limit of $k / a H \rightarrow 0$ is

$$
u_{k} \propto z
$$

Eq.(6) indicates that $P_{S}(k, a)$ becomes approximatly constant for mode $k$ to be well outside the Hubble radius, which can also be seen directly from the equation of motion of $\mathcal{R}_{k}$ :

$$
\dot{\mathcal{R}}_{k}=\frac{2}{3}(1+w)^{-1} H\left(\frac{k}{a H}\right)^{2} \Psi_{k},
$$

here, $w=p / \rho$ is the ratio of the pressure to the density.

To describe the period of slow-rolling of inflation, one defines two parameters $\epsilon$ and $\delta$

$$
\begin{aligned}
\epsilon & \equiv \frac{-\dot{H}}{H^{2}} \ll 1, \\
\delta & \equiv \frac{\ddot{\phi}}{H \dot{\phi}} \ll 1,
\end{aligned}
$$

in term of which $z^{\prime \prime} / z$ can be expressed as [3]

$$
\frac{z^{\prime \prime}}{z}=2 a^{2} H^{2}\left(1+\frac{3}{2} \delta+\epsilon+\frac{1}{2} \delta^{2}+\frac{1}{2} \epsilon \delta+\frac{1}{2} \frac{1}{H} \dot{\epsilon}+\frac{1}{2} \frac{1}{H} \dot{\delta}\right)
$$

and the conformal time

$$
\eta=\int \frac{d t}{a}=\int \frac{d a}{a^{2} H}=\frac{-1}{a H}+\int \frac{\epsilon d a}{a^{2} H}
$$

Assuming $\epsilon$ and $\delta$ to be constant during the period of slow rolling, Eq.(四) can be reduced to [3]:

$$
u_{k}^{\prime \prime}+\left(k^{2}-\frac{\nu^{2}-\frac{1}{4}}{\eta^{2}}\right) u_{k}=0
$$


where $\nu=(1+\delta+\epsilon) /(1-\epsilon)+1 / 2$ and

$$
\eta=\frac{-1}{a H}\left(\frac{1}{1-\epsilon}\right) .
$$

We emphasize that to obtain Eqs.(11) and (12), one has neglected the terms proportional to $\dot{\epsilon}$ or $\dot{\delta}$ in Eq.(9) and taken $\epsilon$ in Eq.(10) as a constant.

The solution of Eq.(11) for $u_{k}$ is

$$
u_{k}=\frac{1}{2} \sqrt{\pi} e^{i\left(\nu+\frac{1}{2}\right) \frac{\pi}{2}}(-\eta)^{\frac{1}{2}} H_{\nu}^{(1)}(-k \eta)
$$

In the limit of $k / a H \rightarrow 0, u_{k}$ becomes

$$
u_{k} \rightarrow e^{i\left(\nu-\frac{1}{2}\right) \frac{\pi}{2}} 2^{\nu-\frac{3}{2}} \frac{\Gamma(\nu)}{\Gamma\left(\frac{3}{2}\right)} \frac{(-k \eta)^{\frac{1}{2}-\nu}}{\sqrt{2 k}} .
$$

Substituting $\eta$ of Eq.(12) into Eq.(14), one has the power spectrum in the slow rolling approximation,

$$
P_{S}^{(S R A)}(k, a)=\frac{2^{2 \nu-3}}{4 \pi^{2}} \frac{\Gamma^{2}(\nu)}{\Gamma^{2}\left(\frac{3}{2}\right)}(1-\epsilon)^{2 \nu-1} \frac{H^{4}}{|\dot{\phi}|^{2}}\left(\frac{k}{a H}\right)^{3-2 \nu} \quad, \quad \text { as } \quad \frac{k}{a H} \rightarrow 0 .
$$

To obtain the final value of power spectrum, what has been done in the literature is to evaluate the time dependent $P_{S}^{(S R A)}(k, a)$ in the equation above at $k=a H$ [2, 3]:

$$
\begin{aligned}
P_{S}^{(S R A)}(k) & \left.\simeq Q(k, a)\right|_{a H=k} \\
& =\left.\frac{2^{2 \nu-3}}{4 \pi^{2}} \frac{\Gamma^{2}(\nu)}{\Gamma^{2}\left(\frac{3}{2}\right)}(1-\epsilon)^{2 \nu-1} \frac{H^{4}}{|\dot{\phi}|^{2}}\right|_{a H=k},
\end{aligned}
$$

where $Q(k, a)$ is defined as the right hand side (R.H.S.) of Eq.(15).

Note that $P_{S}^{(S R A)}(k, a)$ in Eq.(15) is valid only in the limit of $k / a H \rightarrow 0$, however, to obtain Eq.(17) one takes $k / a H=1$. One wonders if the value in Eq.(17) is a good approximation to the asymptotic value $P_{S}(k)$ in the limit of $k / a H \rightarrow 0$ ? Furthermore, $Q(k, a)$ is mathmetically divergent for $\nu>\frac{3}{2}$ and zero for $\nu<\frac{3}{2}$ in the limit of $k / a H \rightarrow 0$, so $Q(k, a)$ can only be a description of the true evolution of $P_{S}(k, a)$ for some specific range of $\ln (a H / k)$. Therefore it is questionable to have the asymptotic value of the real spectrum obtained from Eq.(15). 
In this brief report, we numerically solve Eq.(四) and study the evolution of $Q(k, a)$ and $P_{S}(k, a)$ after horizon crossing. The initial conditions of $u_{k}$ and $u_{k}^{\prime}$ are given by Eq.(13) when the mode $k$ crosses out the Hubble radius. In the numerical calculation of the amplitude of the primordial spectrum we take two models as examples: one is chaotic inflation model with a potential $V=m^{2} \phi^{2} / 2$, and the other is natural inflation model [7] $V=\Lambda^{4}[1+\cos (\phi / f)]$. We consider only the amplitude of the primodial spectrum for the COBE scale $\left(k_{C O B E} \simeq\right.$ 7.5 $\left.a_{0} H_{0}\right)$. The fluctuation mode corresponding to this scale crosses out the Hubble radius at the time e-folds $N \simeq 57$ before the end of inflation. This condition determines the value of inflaton field $\left.\phi\right|_{a H=k_{C O B E}}$. Our numerical results on the evolution of $P_{S}\left(k_{C O B E}, a\right)$ and $Q\left(k_{C O B E}, a\right)$ are presented in Fig.1 and Fig.2. In each of these figures, a dashed horizontal line marks the exact asymptotic value of the power spectrum.

Fig.1(a) and Fig.2(a) show the evolution of $P_{S}\left(k_{C O B E}, a\right)$ after the COBE scale crosses outside the horizon during inflation. From these two figures one can see that when $\ln \left(a H / k_{C O B E}\right) \approx 3, P_{S}\left(k_{C O B E}, a\right)$ starts to be approximatly a constant. In Fig.3 and Fig.4 we show the relative error between $Q\left(k_{C O B E}, a\right)$ and the exact numerical result of $P_{S}\left(k_{C O B E}, a\right)$ when $\ln \left(a H / k_{C O B E}\right) \geq 3$. For $3 \leq \ln \left(a H / k_{C O B E}\right) \leq 6$ it is very small, so $Q\left(k_{C O B E}, a\right)$ in this range of $\ln \left(a H / k_{C O B E}\right)$, which we denote as $\left.Q\left(k_{C O B E}, a\right)\right|_{\dot{\mathcal{R}} \simeq 0}$, will be a good approximation to the real spectrum $P_{S}\left(k_{C O B E}, a\right)$ in the same range, and then can be taken reasonablly as the asymptotic value of the spectrum in the limit of $k / a H \rightarrow 0$.

Fig.1(b) and Fig.2(b) show the evolution of $Q\left(k_{C O B E}, a\right)$ after crossing outside the horizon. From these figures, we can see that the uncertainty in calculating $\left.Q\left(k_{C O B E}, a\right)\right|_{\dot{\mathcal{R}} \simeq 0}$ by using the approximate formula Eq.(17) is of order 1\%. We conclude that the usual analytical treatment in obtaining the primordial power spectrum is feasible with a good accuracy.

Before concluding this report, we should point out that Nakamura and Stewart [8] have tried to tackle this issue by expanding analytically the R.H.S. of Eq.(15) at $k=a H$ to the first-order term of $\ln (a H / k)$. However, as shown in Fig.1 and Fig.2, when $P_{S}\left(k_{C O B E}, a\right)$ starts to be approximately a constant, $\ln \left(a H / k_{C O B E}\right)$ is larger than about 3 . This raises another question about the validity of the analytical expansion, which can not be answered 
by an analytical argument. So a numerical study presented in this paper is necessary.

We thank R.H. Brandenberger and D. Lyth for reading the manuscript and comments. We also thank Y.S. Piao and Z.-H. Lin for discussions. This work was supported in part by the National Natural Science Foundation of China. 


\section{REFERENCES}

[1] For a review, see D.H. Lyth and A. Riotto, Phys. Rep. 314(1999) 1.

[2] D.H. Lyth and E.D. Stewart, Phys. Lett. B274 (1992) 168.

[3] E.D. Stewart and D.H. Lyth, Phys. Lett. B302 (1993) 171.

[4] J.M. Bardeen, Phys. Rev. D22 (1980) 1882.

[5] J.E. Lidsey, A.R. Liddle, E.W. Kolb and E.J. Copeland, Rev. Mod. Phys. 69 (1997) 373.

[6] V.F. Mukhanov, H.A. Feldman and R.H. Brandenberger, Phys. Rep. 215 (1992) 203.

[7] F.C. Adams, J. R. Bond, K. Freese, J. Frieman, and A. Olinto, Phys. Rev. D47 (1993) 426.

[8] T.T. Nakamura and E.D. Stewart, Phys. Lett. B381 (1996) 413. 


\section{FIGURES}
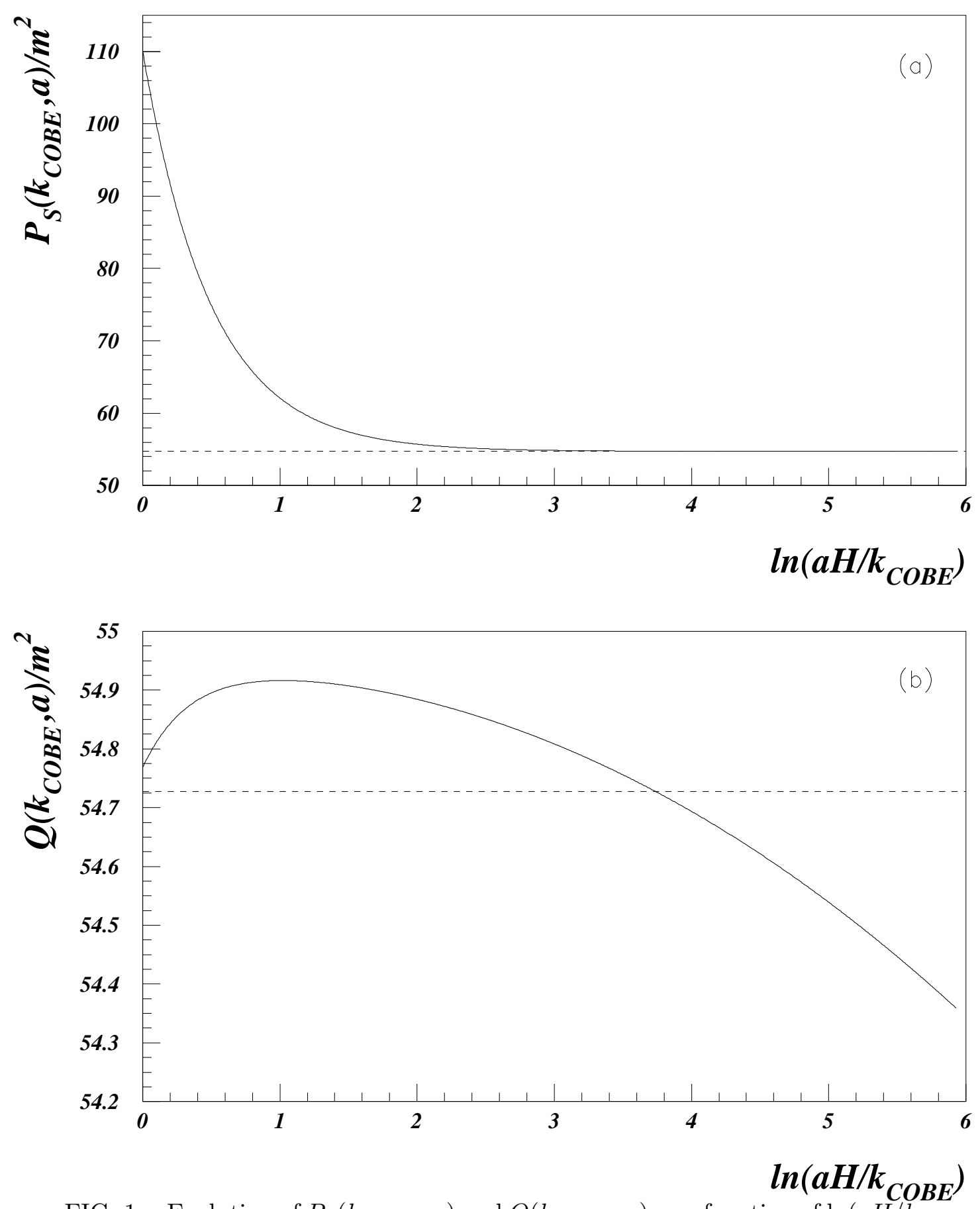

FIG. 1. Evolution of $P_{S}\left(k_{C O B E}, a\right)$ and $Q\left(k_{C O B E}, a\right)$ as a function of $\ln \left(a H / k_{C O B E}\right)$ for model $V(\phi)=m^{2} \phi^{2} / 2$ 

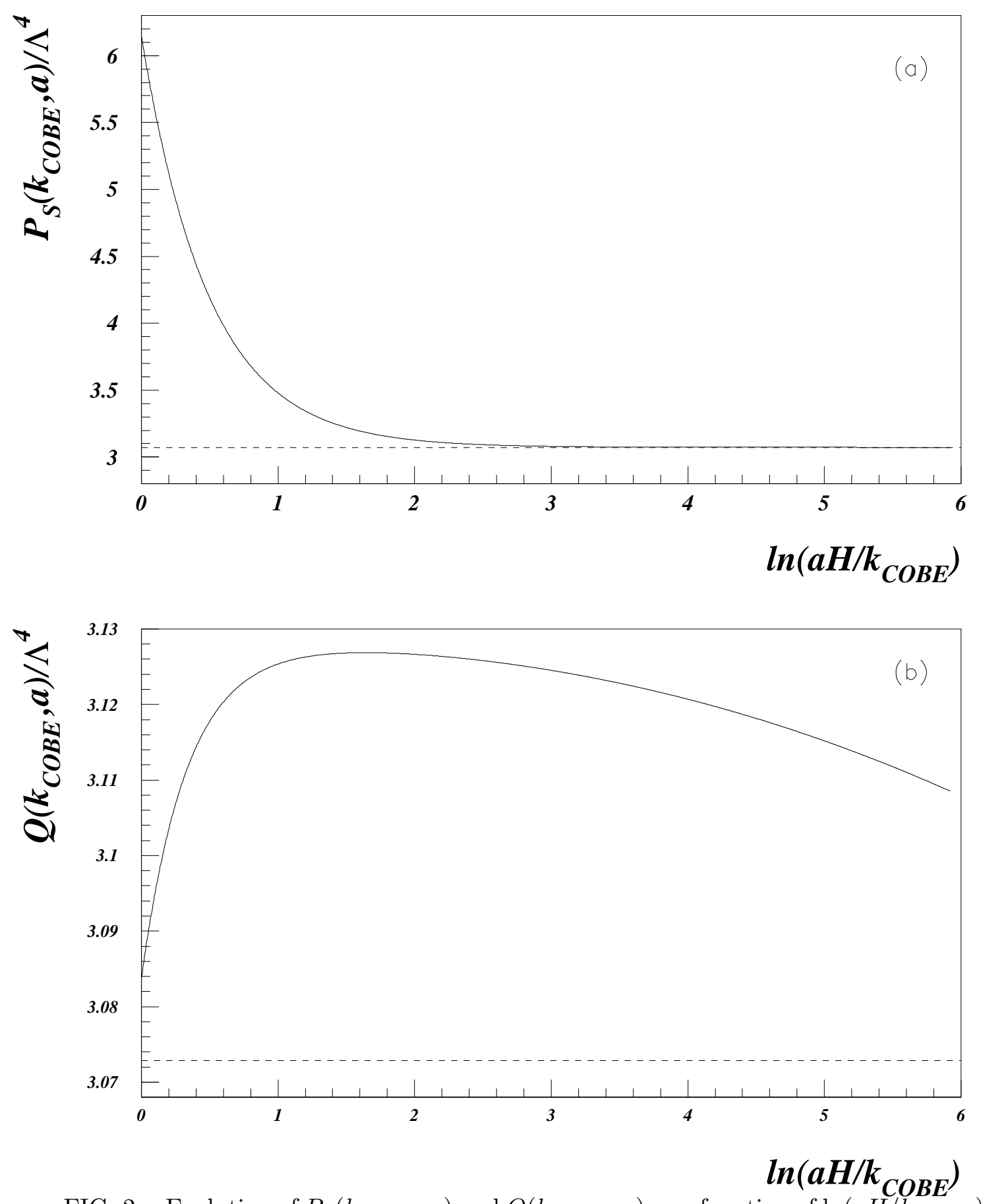

FIG. 2. Evolution of $P_{S}\left(k_{C O B E}, a\right)$ and $Q\left(k_{C O B E}, a\right)$ as a function of $\ln \left(a H / k_{C O B E}\right)$ for model $V=\Lambda^{4}[1+\cos (\phi / f)]$ with $f=1 m_{p l}$. 


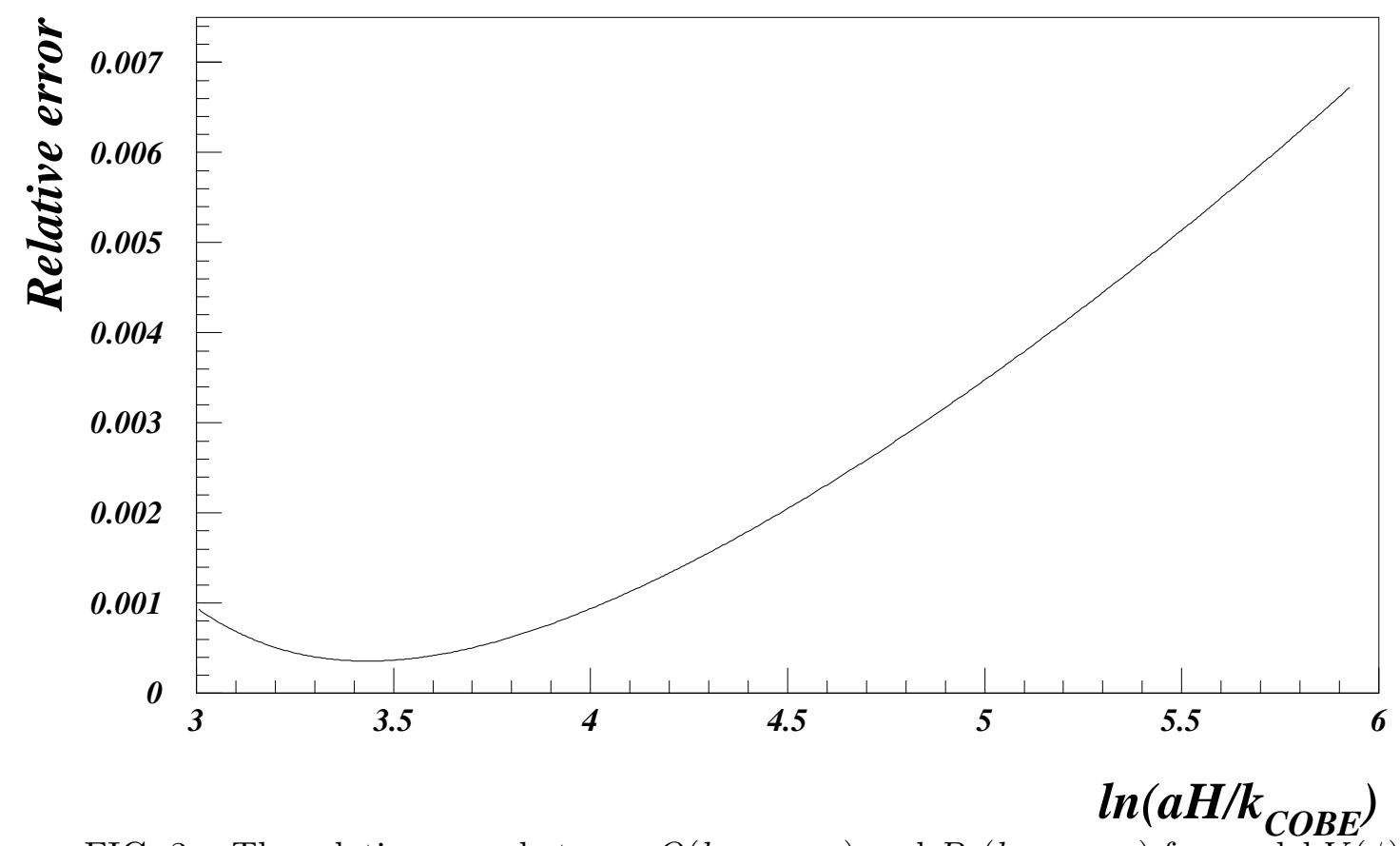

FIG. 3. The relative error between $Q\left(k_{C O B E}, a\right)$ and $P_{S}\left(k_{C O B E}, a\right)$ for model $V(\phi)=m^{2} \phi^{2} / 2$

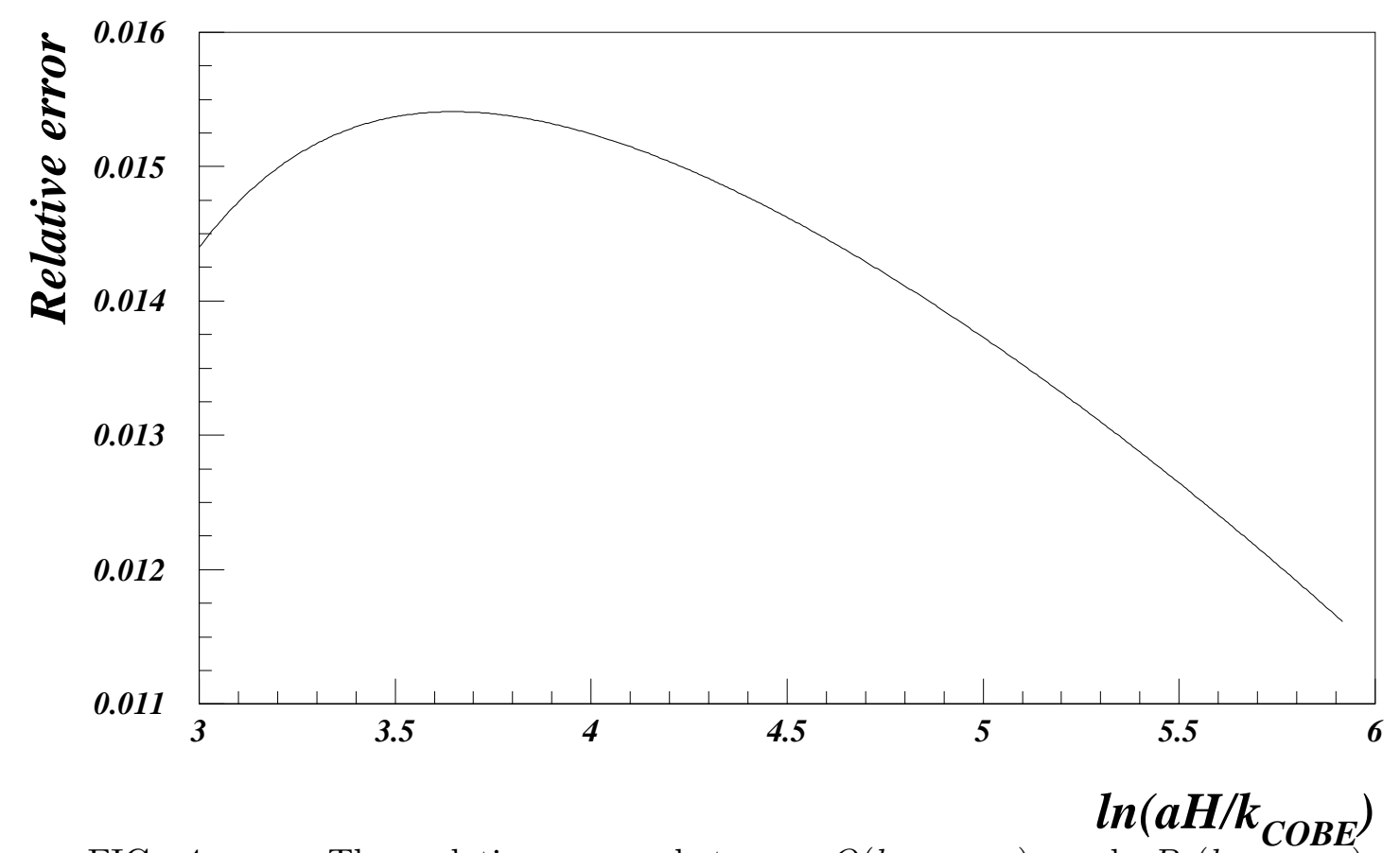

FIG. 4. The relative error between $Q\left(k_{C O B E}, a\right)$ and $P_{S}\left(k_{C O B E}, a\right)$ for model $V=\Lambda^{4}[1+\cos (\phi / f)]$ with $f=1 m_{p l}$. 